\title{
Befähigung zu gesellschaftlicher und politischer Verantwortungsübernahme als Teil Globalen Lernens - Was heißt hier Neutralität?
}

\begin{abstract}
Zusammenfassung
Bildung für nachhaltige Entwicklung und insbesondere Globales Lernen als politische Bildung bieten vielfältige thematische Möglichkeiten, um politische Lern- und Bildungsprozesse gestalten und anregen zu können. In der schulischen Praxis bleibt das Potenzial teilweise ungenutzt, da bei Lehrpersonen eine große Unsicherheit herrscht, ab wann die Auseinandersetzung mit Themen des Globalen Lernens „politisch“ wird und damit vermeintlich als nicht ",neutral“, sondern unter Umständen als überwältigend gelten kann. Die Forderung nach Neutralität, die an Lehrpersonen herangetragen, aber auch von (angehenden) Lehrpersonen selbst als Teil ihrer pädagogischen Professionalität übernommen wird, führt dazu, dass der Fokus in der Vorbereitung auf die Bildungspraxis auf „nicht kontroverse" ökologische Gegebenheiten gelegt wird. Der Anspruch eine professionelle, d. h. „neutrale“ Lehrkraft zu sein, scheint mit der Thematisierung politisch gewachsener Strukturen und globaler Ungerechtigkeiten in Konflikt zu stehen, die für Globales Lernen zentral sind. Dabei spielt es eine Rolle, dass die Frage nach der Normativität der Konzepte wenig reflektiert wird. Der Beitrag geht mithilfe eines empirischen Forschungssettings der Frage nach, wie sich das professionelle Selbstbild (angehender) Lehrkräfte im Kontext der Debatte um das Politische und die Normativität im Globalen Lernen und deren Bearbeitung in Bezug auf das vermeintliche Neutralitätsgebot gestaltet und welche Herausforderungen dabei für das Feld entstehen.
\end{abstract}

Schlüsselworte: politische Bildung, Globales Lernen, Bildung für nachhaltige Entwicklung, Neutralität, Normativität

\footnotetext{
Abstract

Education for Sustainable Development and in particular Global Learning as political education fundamentally offer a wide range of thematic possibilities for shaping and stimulating political learning and education processes. In school practice, this potential often remains untapped as there is a great deal of uncertainty among teachers as to when the discussion of these topics will become "political" and thus, supposedly, not "neutral" but, under certain circumstances, overwhelming. The demand for neutrality, which is addressed to teachers,
}

but which is also adopted by (prospective) teachers themselves as part of their pedagogical professionalism, leads to a focus in educational practice or in the preparation for this, on "non-controversial" ecological conditions. Addressing politically evolved structures and global injustices, which are central to Global Learning, seems to be in conflict with the claim to be a professional, i.e. "neutral" teacher. Thereby it plays a role that the question of the normativity of the concepts is little reflected. With the help of an empirical research setting, this article explores the question of how the professional self-image of (prospective) teachers is shaped in the context of the debate about the political element and normativity in Global Learning and their treatment in relation to the supposed requirement of neutrality, and which challenges this poses for the area.

Keywords: Citizenship Education, Global Learning, Education for Sustainable Development, Neutrality, Normativity

\section{Einleitung}

Die Forderung nach politischer Neutralität von Lehrpersonen ist im öffentlichen Diskurs prominent geworden und zeigt dort seine Wirkung, auch wenn sie als pädagogisches Qualitätskriterium in den Fachdebatten bereits berechtigterweise zurückgewiesen wurde. Diese Forderung, so unsere These, steht im Widerspruch zu den Aufgaben von Globalem Lernen und einer Bildung für nachhaltige Entwicklung $(\mathrm{BNE})^{1}$, da es dabei um die Auseinandersetzung mit und Veränderungen von nichtnachhaltigen Lebensweisen und globalen Ungleichheiten geht. Ein Neutralitätsverständnis des „Sich-Heraushaltens“ (Nonnenmacher, 2011) steht konträr zu den gewünschten transformatorischen Bildungsprozessen (UN, 2015), für die vielmehr eine politisch-interessierte Lehrkraft als Vorbild nötig wäre (Overwien, 2019b).

Der Beitrag zeigt, basierend auf einer qualitativen Interviewstudie, die anhand der dokumentarischen Methode konzipiert und analysiert wurde, wie sich das professionelle Selbstbild (angehender) Lehrkräfte im Kontext der Debatte um das Politische im Globalen Lernen und dessen Bearbeitung in 
Bezug auf das vermeintliche Neutralitätsgebot gestaltet. Sowohl das Globale Lernen als auch die politische Bildung haben generell einen normativen Kern, eine Werteorientierung, die in dem vorliegenden Beitrag nicht zur Disposition gestellt wird. Die rekonstruierten Orientierungen der angehenden Lehrkräfte zeigen hingegen, dass ihnen die Bedeutsamkeit der Normativität eines politischen Globalen Lernens, das die Kritik an globalen Verhältnissen, wie postkoloniale Kontinuitäten, kapitalistischer Wachstumsimperativ oder Paternalismus etc., umfasst, unklar ist. Das Verfolgen des vermeintlichen Neutralitätsgebots kann daher zu einer Vernachlässigung des politisch-normativen Impetus führen. Besonders bei (angehenden) Lehrkräften erzeugt die Herausforderung Globales Lernen zu unterrichten Unsicherheiten, die den Rückgriff auf das vermeintliche Neutralitätsgebot begünstigen und die zu einer Entpolitisierung des Globalen Lernens beitragen können.

Im Zentrum dieses Beitrages steht daher das professionelle Selbstbild angehender Lehrkräfte im Kontext des Globalen Lernens. In Abschnitt 1 wird der Diskurs um die Förderung gesellschaftlicher Verantwortungsübernahme für die Förderung einer nachhaltigeren Entwicklung und daran anschließend die Frage der Normativität und die Forderung nach Neutralität dargestellt. Die analysierten Orientierungen der interviewten Lehrkräfte werden in Abschnitt 2 anhand verschiedener Transkriptausschnitte veranschaulicht. Abschließend (3) werden die Erkenntnisse zu Normativität und dem Politischen im Globalen Lernen als Herausforderungen diskutiert.

\section{Gesellschaftliche Verantwortungsvermitt- lung und Globales Lernen - Normativität und Neutralitätsforderung}

Globales Lernen ist eng verknüpft mit der Übernahme gesellschaftlicher Verantwortung insbesondere im Hinblick auf intra- und intergenerationelle Gerechtigkeit. Das politische Leitbild einer nachhaltigen Entwicklung versucht Bildung zu nutzen, um nachhaltige Lebensweisen, Konsumformen usw. zu etablieren, die als verantwortliches Handeln und gesellschaftliche Verantwortungsübernahme für eine nachhaltige Zukunft definiert werden. Das Konzept BNE ist daher von Beginn an normativ aufgeladen und dient zur Umsetzung der Nachhaltigkeitsbestrebungen. Akteur/-innen des Globalen Lernens haben die Bedeutung des Leitbilds der nachhaltigen Entwicklung mehrfach anerkannt und sich als Teilbereich einer BNE verortet (Schreiber, 2012, S. 29).

Gleichzeitig wird die hohe Wert- und Normenorientierung des Leitbildes im Globalen Lernen als Widerspruch zwischen Bildungsanspruch und der impliziten Normativität diskutiert. Asbrand und Scheunpflug weisen darauf hin, dass Lernen zwar angeregt werden kann, Lernprozesse aber nicht determiniert werden können, d. h. dass „Zurückhaltung gegenüber der Annahme geboten [sei], normativ orientierte Bildungsangebote könnten direkt in Lernprozesse im Sinne von Einstellungs- und Verhaltensänderungen" (Asbrand \& Scheunpflug, 2005, S. 475) eingreifen. Normative Zurückhaltung soll so normative „Postulatspädagogik“ (ebd., S. 473) verhindern.

Diese Forderung spielt eine zentrale Rolle, ist sie doch eng verbunden mit „dem Politischen “ auf der einen Seite sowie dem vermeintlichen Neutralitätsgebot auf der anderen Seite: Wie wird Normativität in Bezug auf Globales Lernen diskutiert und welche empirischen Erkenntnisse gibt es? Applis (2014) erforscht dokumentarisch wie wertorientiertes Lernen im Kontext des Globalen Lernens stattfinden kann. Er weist darauf hin, dass Möglichkeiten konstruktiver diskursiver Auseinandersetzungen im Rahmen der Themen des Globalen Lernens benötigt werden, in denen moralrelevante Kompetenzen differenziert betrachtet werden, um eine Sensibilisierung für das Erkennen von Situationen anzuregen, in denen die Bezugnahmen auf ethische Werte relevant ist (Applis, 2014, S. 19). Ähnlich argumentieren auch Anselm et al. (2018), die sich mit zentralen ethischen Fragestellungen des normativen Nachhaltigkeitsdiskures auseinandersetzen, beispielsweise der Frage danach, was ein "guter" oder "nachhaltiger" Lebensstil ist. Seminarveranstaltungen für Lehramtsstudierende werden in „Diskurs-Arenen“ durchgeführt, die sich am Diskursbegriff von Habermas orientieren und der Logik des Einübens von Diskursfähigkeit folgen, um die verschiedenen Standpunkte einer nachhaltigen Entwicklung durch argumentative Reflexion und persönliche Aneignung der normativen Implikationen diskutieren zu können (Anselm et al., 2018, S. 37ff.). Kater-Wettstädt und Asbrand befassen sich mit der Frage, wie Schüler/-innen in Lehr-Lernarrangements des Globalen Lernens mit normativen Handlungsaufforderungen umgehen. Die Ergebnisse ihrer empirischen Studie zeigen, dass Schüler/-innen keine individuelle Handlungsfähigkeit zum Globalen Lernen erwerben, da Handlungsaufforderungen der Lehrer/-innen entweder reproduziert oder von den Schüler/-innen an andere Verantwortliche (die Allgemeinheit, einflussreiche Politiker/-innen) delegiert werden. Wenn globale Zusammenhänge als Probleme thematisiert werden, formulieren Lehrkräfte fast zwangsläufig moralische Appelle und konkrete Handlungsoptionen, die als „richtige“ oder wünschenswerte Problemlösungen kommuniziert werden. Die Schüler/-innen haben kaum eine andere Möglichkeit außer diese Positionen, ohne wirkliche Aneignung, zu reproduzieren. Die Autorinnen weisen darauf hin, dass es für die pädagogische Praxis des Globalen Lernens notwendig ist, auch normative Handlungsmöglichkeiten als kontrovers, hinsichtlich ihrer Chancen und Grenzen, zu diskutieren, den Umgang mit Ungewissheit einzuüben und zu reflektieren, warum ethisch verantwortungsvolles Handeln in Bezug auf globale Problemlagen, in denen es nicht nur singuläre Lösungen gibt, schwierig realisierbar ist (Wettstädt \& Asbrand, 2014, S. 11f.). Kater-Wettstädt stellt aufbauend auf den Ergebnissen der Studie fest, dass die Reflexion der eigenen Standort- bzw. Kulturgebundenheit, die Begrenzung und Steuerung von Erkenntnisprozessen und die Reflexion von Handlungsoptionen bislang zu wenig berücksichtigt werden. Die Autorin schlägt ein verstärktes Philosophieren in Schule und Unterricht vor, um (ergebnis-)offene Prozesse anzuregen und dadurch eine kritisch-reflektierte und konstruktiv-lösungsorientierte Haltung einzuüben. Die Auseinandersetzung mit Fragen, für die es keine eindeutigen oder richtigen Antworten gibt, sei zentral: Das Philosophieren könnte dazu dienen eine konstruktive Haltung gegenüber Unsicherheit und Ungewissheit einzuüben, in deren Rahmen Werte und Normen als Orientierung fungieren und exploriert werden können (Kater-Wettstädt, 2019, S. 132f.). 
Ein weiterer zentraler Diskussionsstrang im Kontext der hier behandelten Thematik stammt aus der politischen Bildung. Hier ist die Frage virulent, ob politische Bildung parteiisch ist, ein normatives Ziel verfolgend, oder ob dies schon überwältigend sei. Diese Debatte führte zum Beutelsbacher Konsens, der die wirkmächtigste Leitlinie pädagogischen Handelns in der politischen Bildung darstellt. In ihm werden das Überwältigungsverbot, das Kontroversitätsgebot und die Schüler/-innenorientierung als Leitlinien politischer Bildung beschrieben (Wehling, 1977). In gewisser Weise plädiert der Beutelsbacher Konsens damit für einen Pluralismus, verstanden als das Abbilden verschiedener, im Diskurs zu einem Thema vertretener Positionen. Seit einigen Jahren gewinnt ein Deutungsmuster und eine politische Strategie an Bedeutung, die das Überwältigungsverbot und das Kontroversitätsgebot des Beutelsbacher Konsens als „Neutralität“ definiert. Lehrer/-innen und politische Bildner/-innen allgemein, die sich, so der Vorwurf, nicht „neutral“ verhielten, indem sie beispielsweise die Positionen einer Partei zu einem politischen Fall oder Sachverhalt kritisch betrachten, werden mit einem Indoktrinationsvorwurf bedacht. Schüler/-innen und Eltern werden sogar dazu aufgefordert, solche Lehrkräfte zu denunzieren (Däuble, 2019). Dies ist kein neues Phänomen, sondern historisch als eine Wirkung des Beutelsbacher Konsens zu beschreiben, die ihm von Anfang an eingeschrieben war (Nonnenmacher, 2011). Obwohl vielfach gegen eine solche Lesart des Beutelsbacher Konsens protestiert und diese auch argumentativ angegriffen wurde (Besand, 2020; Lösch, 2020; Reinhardt, 2019; Overwien, 2019b), ist sie doch wirkmächtig, was die noch recht überschaubare empirische Studienlage zeigt (Oberle et al., 2018; Heil, 2020). So wurde in der Diskussion beispielsweise darauf verwiesen, der Beutelsbacher Konsens ziele auf Kontroversität, nicht auf Neutralität (Engartner, 2020, S. 12) und rechtliche Analysen haben die Neutralitätsforderung in Zweifel gezogen (Hufen, 2019; Wrase, 2020). Angehende Lehrkräfte vertreten, so die tatsächliche (s. o.) und die gefühlte Empirie (Nonnenmacher, 2011), häufig die Position, die gute Lehrkraft sei politisch neutral, halte mit der eigenen Position „hinter den Berg“" und lasse möglichst alle Positionen gelten. Dass letztere Position vor dem Hintergrund der diskursiven und realpolitischen Stärkung der radikalen Rechten eine Fehlvorstellung ist, darauf wurde in Diskussionen der politischen Bildung ausführlich hingewiesen (Cremer, 2019; Mittnik et al., 2018). In Bezug auf normative und handlungsorientierte Bildungskonzepte sind Lehrkräfte auch dem Grundgesetz verpflichtet, in dem in Artikel 20a auch die nachhaltige Entwicklung verankert ist. Diese Verpflichtung muss auch gesehen werden, ein alleiniger Bezug auf den Beutelsbacher Konsens scheint hier zu wenig zu sein (Overwien, 2019b).

Eng zusammen mit der Frage nach Normativität hängt also diese nach vermeintlicher Neutralität, die v.a. in der politischen Bildung diskutiert wird. Dabei ist die Annahme leitend, dass das Politische und politische Fragen immer mit Normativität (Was ist gute Politik? Wie wollen wir zusammenleben? usw.) verknüpft sind, weshalb ein politischer Aushandlungsprozess notwendig ist. Globales Lernen ist ein Inhaltsfeld politischer Bildung, das in der schulischen politischen Bildung allerdings zunächst wenig thematisiert wurde (Overwien, 2017). Eine Begründung dafür kann die allgemeine Furcht vor
Überwältigung im Sinne des Beutelsbacher Konsens sein, sowie die Angst vor einer „Katastrophenpädagogik“. Im Rahmen des Globalen Lernens haben sich daher positive Zukunftsvisionen durchgesetzt (Overwien, 2019a, S. 306). Hippe (2016, S. 204ff.) kritisiert, dass diese Vorgehensweise einen Teil der Realität ausblendet, und fordert bei der Thematisierung des Klimawandels eine kritische politische Bildung, um auf den Unterschied zwischen einem symbolpolitischen Klimaschutzdiskurs und dem eigenen Handeln, das zu negativen Veränderungen führen kann, aufmerksam zu machen. Konzepte der kritischen politischen Bildung betonen die Möglichkeit, der reflexiven Selbstpositionierung der Lehrpersonen und fokussieren im Kontext von Globalem Lernen auf die Analyse politischer Konflikte und Probleme, die in diesem Kontext entstehen. Dabei geht es um die strukturelle Analyse unterschiedlicher Interessen, von politischen, ökonomischen und sozialen, globalen wie nationalen Macht- und Herrschaftsverhältnissen und von ideologischen Begründungsmustern etc. (Lösch \& Thimmel, 2010).

Problematisch an der Neutralitätsdiskussion ist zudem, dass das Ziel der politischen Mündigkeit ebenso pluralistisch gedacht wird, in dem Sinne, dass Kontroversität mit Mündigkeit gleichbedeutend ist: Junge Menschen bekommen in Bildungssettings die Gelegenheit, sich eine eigene Meinung zu bilden, sie können aus Positionen wählen, die zumeist von Lehrpersonen ausgewählt werden und die selbst aus dem Rahmen der gesellschaftlich hegemonialen Diskurse stammen. Auch hier gilt politische Zurückhaltung als Tugend, damit Lernende nicht überwältigt werden. Das „Neutralitätsmantra“ ist also eng verknüpft mit einer zweifelhaften Vorstellung einer unpolitischen und neutralen Lehrperson, die sich politisch selbst zurückhält. So zeigt Heil (2020) im Rahmen einer Fragebogenerhebung, dass angehende Lehrpersonen oftmals das Denkmuster reproduzieren, sie seien als Lehrekräfte zu Neutralität verpflichtet und dürften ihre eigene Position nicht offenlegen.

Im Folgenden steht die Frage im Zentrum, wie sich das professionelle Selbstbild (angehender) Lehrkräfte im Kontext der Debatte um das Politische und Normative im Globalen Lernen und dessen Bearbeitung in Bezug auf das vermeintliche Neutralitätsgebot gestaltet.

\section{„Also man soll sich ja mit seinen politischen Meinungen zurückhalten." Empirische Einblicke zum Themenkomplex Globales Lernen, Normativität und Neutralität}

Die im Zentrum des Beitrags stehende qualitativ-rekonstruktive Studie wurde in Baden-Württemberg durchgeführt. Dort ist BNE im aktuellen Bildungsplan eine von sechs Leitperspektiven, die einen ganzheitlichen Ansatz verfolgen, indem BNE fächerübergreifend und spiralcurricular in sämtlichen Schularten unterrichtet werden soll (KM BW, 2016). Das empirische Material wurde mit Hilfe der Dokumentarischen Methode analysiert. Die methodologische Grundlage der Dokumentarischen Methode bezieht sich auf die Wissenssoziologie Mannheims (1980) und der Unterscheidung zwischen kommunikativem (theoretischem, explizitem) und konjunktivem (implizitem, handlungsleitendem) Wissen. Die Erhebung fand im Rahmen von fünf Workshops an einem außerschulischen Lern- 
ort für Globales Lernen statt, an dem Workshops, Stadtrundgänge und Veranstaltungen für Schulklassen, Studierende, Referendar/-innen und Weiterbildungen für Lehrkräfte angeboten werden. Insgesamt wurden 17 leitfadengestützte Einzelinterviews mit zwölf Referendar/-innen und fünf Studierenden geführt (zwölf weibliche und fünf männliche Teilnehmende), aus dem Grundschul-, Haupt- und Realschullehramt sowie der Sonderpädagogik, die an einem Weiterbildungsworkshops zu Globalem Lernen teilgenommen haben. Die Fragen des Leitfadens wurden möglichst erzählgenerierend formuliert und dienten einer vergleichenden narrativen Ausgestaltung des Interviews (Nohl, 2006, S. 22). Die dokumentarische Interpretation der Interviews erfolgte anhand der formulierenden und reflektierenden Interpretation (für den Zugang zu beiden Wissensebenen) und einer konsequenten sequenzanalytischen und einer (fallinternen und fallexternen) komparativen Analyse zur Bestimmung des dokumentarischen Sinngehalts (Nohl, 2006; Bohnsack, 2014). Die rekonstruierten Orientierungen der interviewten angehenden Lehrkräfte werden im Folgenden anhand ausgewählter Transkriptausschnitte expliziert.

\section{Professionelles Selbstbild und theoretisches Wissen: Bildungspraxis}

Im Interviewmaterial lässt sich als Orientierung der Interviewten rekonstruieren, dass sie über theoretisches Wissen hinsichtlich globaler Probleme und den damit zusammenhängenden Herausforderungen verfügen, gleichzeitig aber über wenig erfahrungsbasiertes Wissen in Bezug auf didaktische Umsetzungsmöglichkeiten. Die folgenden Transkriptauszüge dienen als Veranschaulichung für die rekonstruierte Orientierung. Auf die Relevanz von Bildung für die angestrebte Mündigkeit der Schüler/-innen wird vielfach verwiesen: „[D]ieses Ziel von Bildung is äh immer der mündige Bürger und im Kontext der Globalisierung ähm ist man eben mündig, wenn man mit der Globalisierung klarkommt; des heißt, man muss bewusstmachen, [...] die Kontroversen immer wieder aufzeigen, und [...] Schüler darauf vorbereiten mit diesen Möglichkeiten und aber auch mit den Gefahren ähm zurecht zu kommen." (Max, 108-115)² Globalisierung wird problemzentriert beschrieben, indem explizit auf die Gefahren (siehe auch Wettstädt \& Asbrand, 2014) hingewiesen wird, was sich als implizite Unsicherheit im Umgang mit der Thematik vielfach im analysierten Material zeigt. Eine Studentin beschreibt die generelle Wichtigkeit einer strukturellen Betrachtungsweise und deren stärkere Betonung, um der Komplexität und den Verflechtungen im Bereich nachhaltige Entwicklung gerecht zu werden. Daran anknüpfend betont sie die Möglichkeiten der Veränderung durch Mitbestimmung in der Demokratie, die aber in aktuellen Bildungskonzepten im Hintergrund stehe. Sie verbindet dadurch zwei wesentliche Aspekte: Die strukturelle Betrachtung von Problemen im Kontext des Globalen Lernens und der Erkenntnis, dass alles Gesellschaftliche ein Gewordenes und dadurch auch veränderbar ist: „[E]s muss alles [...] im Bildungskontext, die Probleme mehr aufgegriffen werden und die Vernetzung von den ganzen Problemen. Also sozusagen, dass das alles auch miteinander zusammenhängt und sich die Strukturen und Gesellschaft klar zu machen, allein die Demokratie sich bewusstmachen, dass man wirklich was dagegen tun könnte, dass man in seiner eigenen Gesellschaft was verändern könnte, kommt nicht so durch, würde ich sagen, im Kontext der Bildung." (Mia, 115-125) Auch hier zeigt sich eine Verankerung von theoretischen Wissensbeständen, die als erfahrungsbasiertes Wissen in einer handlungspraktischen schulischen Umsetzung nicht angewendet werden können. Eine Referendarin spricht die Wichtigkeit der Perspektivübernahme an, der einer der zentralen Punkte für Globales Lernen ist. „[D] ie Perspektivübernahme heißt ja irgendwie dann auch n Verständnis für ähm Länder oder Kulturen zu entwickeln und herzustellen, was halt eben auch ganz arg wichtig ist, wenn man irgendwie global handeln möchte." (Lea, 253-259) Das explizite theoretische Wissen verweist darauf, dass Themen des Globalen Lernens relevant sind. Dieses Wissen steht jedoch in Verbindung mit der Unsicherheit bezüglich des Umgangs mit Themen des Globalen Lernens und der Vagheit der Beschreibung, dass man „irgendwie“ global handeln möchte. Wie dieses theoretische Wissen didaktisch aufbereitet und in der Bildungspraxis umgesetzt werden kann, wird von den Interviewten nicht konkret benannt. Lediglich der Verweis auf Veränderung von Konsumpraktiken, mit der Betonung individueller Handlungsmöglichkeiten (z. B. die Anregung zum Kauf ohne Plastiktüten), wird thematisiert (Weselek \& Wohnig, 2020), da diese als nicht überwältigend und somit als neutral gelten.

\section{Professionelles Selbstbild, politische Themen und vermeintliche Neutralitätsanforderung}

In den analysierten Interviewpassagen zeigt sich ein weiterer inhaltlicher Schwerpunkt der aufgeworfenen Orientierung bezüglich des Vorhandenseins theoretischer Wissensbestände, kombiniert mit einer gewissen Uneindeutigkeit bezüglich des didaktischen Umgangs: die prinzipielle Unsicherheit, ob Lehrkräfte politisch sind. „Also man soll sich ja mit seinen politischen Meinungen zurückhalten“ (Ella, 203-204), sagt z.B. eine der Referendarinnen. Einige der angehenden Lehrkräfte gehen davon aus, dass es ihnen offiziell verboten sei, in der Schule politisch zu sein, da sie dadurch die Schüler/-innen überwältigen würden: „[...] ich würde sogar sagen, dass ich als Lehrkraft NICHT so politisch sein DARF. Also in gewisser Weise natürlich eine Meinung haben darf, die aber nur begrenzt vertreten wollen würde, weil ich sonst, ähm, die Schüler vielleicht überwältigen würde und [...] in ihrem Meinungsbildungsprozess auch einschränken würde." (Jan, 110-116) In den Interviewpassagen zeigen sich implizite Verankerungen von Wissensbeständen eines konträren Verhältnisses von „politisch-Sein " und Schule, die sich als diffuse Vorgaben verankert haben, indem das Wissen darüber verinnerlicht ist, dass man nicht „politisch“ sein darf (oder sollte). Es erfolgt aber keine nähere Beschreibung oder Differenzierung zwischen partei-politisch und politisch. Das professionelle Selbstbild ist daran orientiert, auf keinen Fall indoktrinieren zu wollen, oder etwas „falsch“ zu machen: „[...] ich darf ja auch nicht indoktrinieren, deswegen [...] politisch äh kann ich insofern sein, dass ich zum Nachdenken anrege." (Max, 154-156) Die ausgewählten Interviewausschnitte zeigen die implizite Vorstellung der angehenden Lehrkräfte, dass sich Lehrpersonen im schulischen Kontext weder politisch verhalten „sollen“ noch „dürfen“, um die Meinungsbildung der Schüler/-innen nicht einzuschrän- 
ken. Die Unsicherheit und Uneindeutigkeit darüber, was aber genau dieses „politische Handeln“ ist und wo es anfängt, zeigt sich im gesamten Interviewmaterial. Dieser Unsicherheit begegnen die meisten der angehenden interviewten Lehrkräfte dahingehend, indem sie auf die Notwendigkeit verweisen, Neutralität in politischen Debatten zu wahren, um dem Anspruch nach einer kontroversen Behandlung eines politischen Themas gerecht zu werden. Dabei zeigt sich im Material, dass die angehenden Lehrpersonen Kontroversität als ein Abbilden und Nebeneinanderstellen aller Positionen exemplifizieren, was wiederum als neutral gilt und damit der Vorstellung ihres (künftigen) professionellen Lehrkärftehandelns entspricht. Neutralität wird implizit als „,richtiges“ Verhalten bezüglich des professionellen Selbstbildes verstanden. Performativ wird von den Interviewten der Ausdruck einer sachlichen Diskussion verwendet. Dies verweist implizit auf eine (wert)neutrale Diskussion, was als „richtiges“ Professionalisierungsverständnis von den angehenden Lehrkräften verinnerlicht ist: Wer „sachlich“ bleibt, ist professionell. Dabei wird Fachlichkeit von den Interviewten als Gegenpol zu Meinungen beschrieben: „[A]lso mit Nachdenken mein ich eben, dass ich äh die aktuellen Weltgeschehnisse im Bereich der Nachhaltigkeit aufzeige, ähm mit den Schülern kritisch [...] beleuchte, [...] ich möcht nicht meine eigene MEINUNG vermitteln, sondern ich möchte FACHLICH äh angehen." (Tilo, 162-175) Die Unsicherheit unprofessionell zu sein, wird dahingehend beschrieben, dass Lehrpersonen ihre politischen Meinungen zurückhalten müssten und kein politisches Engagement zeigen oder anregen dürften.

$\mathrm{Ob}$ und wie politische Meinungen im schulischen Kontext eingebracht werden können bzw. angemessen sind, wird häufig thematisiert. Im Material wird von den Interviewten, hinsichtlich der politischen Themen des Globalen Lernens, eine interessante Ausnahme formuliert: „Ähm also politisch Partei ergreifen, dürfen wir ja offiziell nicht, aber ähm man kann politische Meinungen auch gerade was jetzt die Umwelt betrifft auch so vertreten, dass man eben nicht ähm die Parteien erwähnt, von daher finde ich schon, dass wir auch politisch ähm fungieren ohne halt jetzt eine Meinungsmache zu verbreiten; also da muss man eben das gute Zwischenmaß finden, ohne jetzt jemandem auf den Schlips zu treten oder irgendwelche, ja Meinungen zu verbreiten." (Lara, 193-204) Politisch können Lehrpersonen nicht sein, indem sie die Positionen von politischen Parteien als Organisationen stärken, sehr wohl aber, wenn sie sich zum Umweltthemen äußern. Hier kommt zum einen das tatsächliche Verbot der aktiven Bevorzugung einer politischen Partei für Beamt/-innen zum Ausdruck (Hufen, 2019), zum anderen aber auch die Überzeugung, Meinungsäußerungen hinsichtlich Umweltthemen seien weniger politisch überwältigend als andere.

Die Ambivalenz der Frage, ab wann ein Thema „politisch“ ist, zeigt sich auch im Hinblick auf den Vergleich von Umweltthemen und dem Thema „Flucht“: „Ich find des Thema Umwelt [...] einfacher, weil ich selber in meinem Alltag [...] auch was selber TUN kann, also ich kann meinen Konsum anschauen[. I]n Bezug auf das Flüchtlingsthema empfind ichs $\mathrm{n}$ bisschen schwieriger, weil da halt natürlich auch sehr viel ähm politisch geregelt wird ähm von oben, [...] ich kann natürlich als Einzelne auch was dafür tun, dass die Immigration von den
Flüchtlingen oder auch von Menschen ausm Ausland besser passiert; [...] und wenn ich jetzt ähm auf die Umwelt schaue, kann ich viel mehr Dinge auch für mich in meinem persönlichen Umfeld tun, ohne dass ich direkt in Kontakt mit anderen Menschen sein muss." (Emma, 102-123) Hier zeigt sich deutlich die bereits oben angesprochene Handlungsstrategie, auf individuelle Konsumpraktiken zurückzugreifen, um sich mit dem Umweltthemen zu befassen. Individuelle Handlungspraktiken werden als durchführbar wahrgenommen und Veränderungen durch persönliches Handeln, im Hinblick auf eine objektivierte Umwelt, als möglich. Eine Lehramtsanwärterin verweist beim Thema Flüchtlinge sehr stark aufden Neutralitätsaspekt: „Also ich finds immer ganz wichtig, dass man da eben NEUTRAL dem gegenüberstellt und alle Aspekte mit angreift, und ähm genau auch alle Meinungen anhört, [...] weil es eben auch sehr emotional gehabt wird und [...] vielleicht auch grad ähm eben Flüchtlinge in der Klasse sind und da muss man sehr vorsichtig mit umgehen, MEINER Meinung nach genau ähm aber eben von allen Seiten neutral betrachten." (Mira, 104-122) Es zeigt sich auch die Unsicherheit, das Thema Flucht didaktisch aufbereiten zu können und die Angst „falsch“ zu handeln sowie die verinnerlichte Positionierung neutral sein zu wollen, die als Anker für „richtiges“ Handeln gilt. Die Referendarin wünscht sich noch weitere Praxisbeispiele und didaktische Umsetzungsmöglichkeiten im Studium, vor allem im Bereich der Flüchtlingsdiskussion. „Also zum Beispiel [...] die Flüchtlingskrise, dass man genau sagt $[, \ldots]$ so leitet man ne neutrale Diskussion ein oder [...] einfach Praxisbeispiele oder wie man handelt, wenn das mal in die Hose geht, es kann ja passieren, dass da Äußerungen, wie ich damit umgeh, erkläre und des wieder in die richtige Richtung lenk sozusagen, dass eben neutral diskutiert wird und nicht durch also auf der emotionalen Ebene." (Mira, 426-437) Der Ausschnitt zeigt eine Bündelung der wahrgenommenen Schwierigkeiten, die hinter den impliziten Vorstellungen, Ängsten oder Unsicherheiten der Interviewten stehen: Was ist, wenn ein Thema „emotional“ und nicht sachlich ist, was kann man tun, wenn man es doch „falsch“ gemacht hat und wie sieht der genau absteckte Raum, was man darf und was nicht, aus? Im Hinblick auf das professionelle Selbstbild lässt sich zusammenfassend die rekonstruierte Diskrepanz zwischen dem Wissen über strukturelle Zusammenhänge und deren Bearbeitung in der Bildungspraxis als Orientierung für eine überwiegende Mehrheit der analysierten Fälle festhalten, die sich insbesondere auch hinsichtlich einer großen Unsicherheit und eines spürbaren Unbehagens dahingehend zeigt, was im Rahmen politischer Themen/Bildung „erlaubt“ und was „verboten“ ist.

\section{Normativität und das Politische - Herausforderungen für das Globale Lernen}

Die theoretischen und empirischen Perspektiven zeigen, dass bezüglich des professionellen Selbstbildes angehender Lehrkräfte die Gefahr besteht, Globales Lernen als etwas außerhalb „des Politischen stehenden“ zu definieren und damit zu entpolitisieren. Dadurch wird ein normatives Konzept, das in seiner Normativität auch hinsichtlich der daraus folgenden Bildungsaufforderungen reflektiert werden müsste, auf die Praxis der moralischen Verhaltensaufforderungen verkürzt. Sämtliche 
Ungleichheitsdimensionen, die die strukturelle und somit politische Ebene betreffen, können so nicht ausreichend behandelt werden, insbesondere die globalen Verflechtungen und Verantwortlichkeiten. Lernarrangements sollten das gewünschte Verhalten nicht steuern oder einfordern, die komplexen Gegenstände Globalen Lernens können hingegen kontrovers und multiperspektivisch thematisiert werden, um die Reflexionsund Urteilskompetenz bei Lernenden zu fördern (Scheunpflug, 2016, S. 34).

Ein Ausblenden von Kontroversen und Widersprüchlichkeiten zeigt sich in den Vorstellungen von angehenden Lehrpersonen, dass Neutralität ein zentraler Aspekt professionellen pädagogischen und didaktischen Handelns sei. Im Material wird in verschiedenen Fällen deutlich, dass angehende Lehrpersonen Kontroversität als das Abbilden und Nebeneinanderstellen aller Positionen beschreiben und dieses Vorgehen als „neutral“ bezeichnen. Dadurch stellt sich u.a. für die Lehrkräfte die Aufgabe, die vorhandenen Vorstellungen von Kontroversität in dem Sinne zu erweitern, dass kritische und normative Vorstellungen als reflektierende politische, gesellschaftliche und ökonomische Urteilsbildungsprozesse stattfinden können und eine adäquate kontroverse Auseinandersetzung mit dem Gegenstand des Bildungsprozesses erfolgt. Die allerdings hegemonial erhobene Forderung nach Neutralität steht im Widerspruch zu den Aufgaben von Globalem Lernen, da die vielfältigen ökologischen, ökonomischen und sozialen Herausforderungen für eine nachhaltige Entwicklung berücksichtigt werden müssen, deren Inhalte letztlich politisch und damit normativ sind und kritische politische Auseinandersetzungen benötigen.

Eine politische Auseinandersetzung mit Themen des Globalen Lernens kann der Gefahr entgegenwirken, dass diese ihren normativen und politischen Kern verlieren, eine (politische) Reflexion der bestehenden (welt-)gesellschaftlichen Verhältnisse fördern und strukturelle Ursachen für globale Ungleichheits- sowie Macht- und Herrschaftsverhältnisse sichtbar machen. Auf einer solchen analytischen Basis ließe sich auch folgende Diskrepanz reflektieren: Angestrebtes professionelles Selbstverständnis, das politische Positionierung innerhalb der Themen des Globalen Lernens auf der einen Seite und Aushalten von Unsicherheit bezüglich einer „Lösung“ der Probleme auf der anderen Seite einfordert. BNE und Globales Lernen sind somit auch als Teil eines emanzipatorischen Bildungsverständnisses definiert, das strukturelle politische, gesellschaftliche, ökologische und ökonomische Ursachen für Probleme und Konflikte in den Blick nimmt und Veränderungen hinsichtlich einen Mehr an Demokratie, Gleichheit, Emanzipation und Schutz der Ökosphäre anstrebt. Die Quelle emanzipatorischer Bildung liegt dabei im Bildungsbegriff selbst, der immer auch die Möglichkeit der Befreiung miteinschließt. Dabei sollte beispielsweise aber auch ein Spannungsfeld des Globales Lernens reflektiert werden, einerseits durch helfendes Engagement zur Überwindung von „Unterentwicklung“ beitragen zu wollen und andererseits die Gefahr zu sehen, dass dieses Engagement auch Reproduktion von Paternalismus oder Übernahme von Stereotypen fördern kann (Scheunpflug, 2016, S. 35).

Die Ergebnisse der Studie führen zu folgenden Fragen: Wie kann der affirmativen Befolgung eines vermeintlichen
Neutralitätsgebotes in den Vorstellungen der (angehenden) Lehrpersonen und der Bildungspraxis im Globalen Lernen eine erfahrungsbasierte Auseinandersetzung und ein reflexiver Umgang mit normativen Auf- und Anforderungen sowie Bildungsinhalten entgegengesetzt werden, in der auch die eigene selbstreflexive politische Positionierung und Fähigkeit der eigenen normativen Begründung gefördert wird? Wie können erfahrungsbasierte Handlungspraktiken im Kontext einer kritischen politischen Bildung für angehende Lehrkräfte erfahrbar gemacht werden, damit sie sich befähigt fühlen, kritische politische Bildungsprozesse im Globalen Lernen auch für Schüler/-innen anleiten zu können? Wie kann der theoretischen Unsicherheit, was im Schulkontext „erlaubt“ ist, begegnet und wie können Lehrkräfte auf dem Weg zu einem politischen, die Normativität reflektierenden Globalen Lernen unterstützt werden? Erste Antworten sind in der Forschung formuliert worden, an die anzuknüpfen wäre. Für die ersten beiden Fragen bietet der Orientierungsrahmen für den Lernbereich Globale Entwicklung Anhaltspunkte (KMK, 2016). Zu der letzten Frage haben Mittnik et al. (2018) in einer Broschüre klare Richtlinien formuliert, was politische Bildung darf.

\section{Anmerkungen}

1 Wir verstehen Globales Lernen, neben der Umweltbildung, als eine der tragenden Säulen einer Bildung für nachhaltigen Entwicklung. Gleichzeitig ist eine klare Feldzuordnung oder Hierarchisierung der Konzepte schwer umsetzbar und auch umstritten. Wir sind uns der Kontroverse um verschiedene Lesarten bewusst, schreiben jedoch im folgenden Text zur Verbesserung der Lesbarkeit von Globalem Lernen. Damit soll gleichzeitig BNE mitbezeichnet sein, was jedoch keine Gleichsetzung oder Entleerung der Begriffe und Konzepte bedeuten soll.

2 Die Namen der Interviewten wurden anonymisiert. Mia und Jan studieren zu dem Zeitpunkt des Interviews noch, die anderen Teilnehmenden befinden sich im Referendariat.

\section{Literatur}

Anselm, S., Hoiß, C. \& Vogt, M. (2018). DISKURS-ARENA: Nachhaltigkeit und Ethik in der Lehrerbildung. In W. Leal Filho (Hrsg.), Nachhaltigkeit in der Lehre: Eine Herausforderung für Hochschulen (S. 37-49). Berlin: Springer. https://doi. org/10.1007/978-3-662-56386-1_3

Applis, S. (2014). Die dokumentarische Methode als Forschungsansatz für die Geographiedidaktik im Bereich des wertorientierten und Globalen Lernens. Zeitschrift für internationale Bildungsforschung und Entwicklungspädagogik, 37(1), 13-20.

Asbrand, B. \& Scheunpflug, A. (2005). Globales Lernen. In W. Sander (Hrsg.), Handbuch politische Bildung, (S. 469-484). Schwalbach/Ts: Wochenschau.

Besand, A. (2020). Politische Bildung unter Druck. Zum Umgang mit Rechtspopulismus in der Institution Schule. Aus Politik und Zeitgeschichte, 14-15, 4-9.

Bohnsack, R. (2014). Rekonstruktive Sozialforschung. Einführung in qualitative Methoden (9., überarb. und erw. Aufl.) Opladen: Farmington Hills.

Cremer, H. (2019). Das Neutralitätsgebot in der Bildung. Berlin: Deutsches Institut für Menschenrechte.

Däuble, H. (2019). In Zeiten digitaler Meldeplattformen - Der Angriff auf demokratische (politische) Bildung und liberales Schulsystem. Gesellschaft - Wirtschaft Politik (GWP), 68(4), 523-534. https://doi.org/10.3224/gwp.v68i4.08

Engartner, T. (2020). Politische Bildung als Verfassungsvoraussetzung. Oder: pädagogische An- und Überforderungen in Zeiten des Rechtspopulismus. Der Staat, 95, 1-27. https://doi.org/10.3790/staa.59.1.117

Heil, M. (2020). Das Lehramt als politischer Beruf. Schriftenreihe POILIS - politische und soziologische Bildung (Band 1). Siegen: universi.

Hippe, T. (2016). Herausforderung Klimaschutzpolitik. Probleme, Lösungsstrategien und Kontroversen. Opladen, Berlin, Toronto: Barbara Budrich. https://doi.org/10.23 07/j.ctvddzj72

Hufen, F. (2019). Politische Jugendbildung und Neutralitätsgebot. Demokratie gegen Menschenfeindlichkeit, 1, 39-46. 
Kater-Wettstädt, L. (2019). Nahtstellen nachhaltigen globalen Lernens - Philosophieren als (neue) Aufgabe von Unterricht. In I. Clemens, S. Hornberg \& M. Rieckmann (Hrsg.), Bildung und Erziehung im Kontext globaler Transformationen (S. 125-140). Opladen, Berlin, Toronto: Barbara Budrich. https://doi.org/10.2307/ j.ctvm201r8.9

KM BW (2016). Bildungsplan des Gymnasiums Gemeinschaftskunde. Stuttgart.

KMK (2016). Orientierungsrahmen für den Lernbereich Globale Entwicklung im Rahmen einer Bildung für nachhaltige Entwicklung (2., akt. und erw. Aufl.). Bonn.

Lösch, B. (2020). Wie politisch darf und sollte Bildung sein? Die aktuelle Debatte um ,politische Neutralität' aus Sicht einer kritisch-emanzipatorischen politischen Bildung. In C. Gärtner \& J. Herbst (Hrsg.), Kritisch-emanzipatorische Religionspädagogik. Diskurse zwischen Theologie, Pädagogik und Politischer Bildung (S. 383-402). Wiesbaden: Springer VS. https://doi.org/10.1007/978-3-658-28759-7_21

Lösch, B. \& Thimmel, A. (Hrsg.) (2010). Kritische politische Bildung. Ein Handbuch. Schwalbach/Ts: Wochenschau.

Mannheim, K. (1980). Strukturen des Denkens. Frankfurt a. M.: Suhrkamp.

Mittnik, P., Lauss, G. \& Schmid-Heher, S. (2018). Was darfpolitische Bildung? Eine Handreichung für LehrerInnen für den Unterricht in Politischer Bildung. Wien: Pädagogische Hochschule Wien.

Nohl, A.-M. (2006). Interview und dokumentarische Methode. Anleitungen für die Forschungspraxis. Wiesbaden: Springer VS.

Nonnenmacher, F. (2011). Handlungsorientierung und politische Aktion in der schulischen politischen Bildung. Ursprünge, Grenzen und Herausforderungen. In ders. \& B. Widmaier (Hrsg.), Partizipation als Bildungsziel. Politische Aktion in der politischen Bildung (S. 83-99). Schwalbach/ Ts: Wochenschau.

Oberle, M., Ivens, S. \& Leunig, J. (2018). Grenzenlose Toleranz? Lehrervorstellungen zum Beutelsbacher Konsens und dem Umgang mit Extremismus im Unterricht. In L. Möllers \& S. Manzel (Hrsg.), Populismus und politische Bildung (S. 53-61). Frankfurt am Main: Wochenschau.

Overwien, B. (2017). Die Zukunft des Lernens in globaler und nachhaltiger Perspektive. In O. A. Burow \& C. Gallenkamp (Hrsg.), Bildung 2030. Sieben Trends, die die Schule revolutionieren (S. 138-150). Weinheim \& Basel: Beltz.

Overwien, B. (2019a). Umwelt, Klimawandel, Globalisierung - Angst in der politischen Bildung? In A. Besand, B. Overwien \& P. Zorn (Hrsg.), Politische Bildung mit Gefühl (S. 304-318). Bonn: Bundeszentrale für politische Bildung.

Overwien, B. (2019b). Politische Bildung ist nicht neutral. Demokratie gegen Menschenfeindlichkeit, 1, 26-38.

Reinhardt, S. (2019). Jagd auf Lehrer statt Beutelsbacher Konsens. Kommentar zum Portal "Neutrale Schulen" der AfD in Hamburg. Gesellschaft. Wirtschaft. Politik (GWP), 68(1), 13-19. https://doi.org/10.3224/gwp.v68i1.01
Scheunpflug, A. (2016). Entwicklungspolitische Bildung und Globales Lernen. Ein Beitrag zur politischen Bildung. Außerschulische Bildung. Zeitschrift der politischen Jugend- und Erwachsenenbildung, 30-37.

Schreiber, J. (2012). Bildung für nachhaltige Entwicklung. In G. Lang-Wojtasik \& U. Klemm (Hrsg.), Handlexikon Globales Lernen (S. 26-30). Münster \& Ulm: Klemm \& Oelschläger.

United Nations (2015). Transforming our world: the 2030 Agenda for Sustainable Development. Zugriff am 24.04.2020 https://www.un.org/en/development/desa/ population/migration/generalassembly/docs/globalcompact/A_RES_70_1_E.pdf

Wehling, H. (1977). Konsens à la Beutelsbach? S. Schiele \& H. Schneider (Hrsg.), Das Konsensproblem in der politischen Bildung (S. 173-184). Stuttgart: Ernst Klett.

Weselek, J. \& Wohnig, A. (2020). Praxisvorstellungen und -erfahrungen von Studierenden und Referendar/-innen zur Umsetzung von Bildung für nachhaltige Entwicklung in Schule und Unterricht. Zeitschrift Didaktik der Sozialwissenschaften, 72-90.

Wettstädt, L. \& Asbrand, B. (2014). Handeln in der Weltgesellschaft. Zum Umgang mit Handlungsaufforderungen im Unterricht zu Themen des Lernbereichs Globale Entwicklung. Zeitschrift für internationale Bildungsforschung und Entwicklungspädagogik, 37(1), 4-12.

Wrase, M. (2020). Wie politisch dürfen Lehrkräfte sein? Rechtliche Rahmenbedingungen und Perspektiven. Aus Politik und Zeitgeschichte, 14-15, 10-15.

\section{Johanna Weselek}

ist akademische Mitarbeiterin am Lehrstuhl für Geographie und am Heidelberger Zentrum Bildung für nachhaltige Entwicklung, Pädagogische Hochschule Heidelberg. Vorher akademische Mitarbeiterin an der Heidelberg School of Education (Universität und Pädagogische Hochschule Heidelberg). Eingereichte Dissertation im Fach Soziologie zum Thema Bildung für nachhaltige Entwicklung.

\section{Alexander Wohnig}

Dr., Juniorprofessor für Didaktik der Sozialwissenschaft an der Philosophischen Fakultät der Universität Siegen. Promotion im Fach Didaktik der Sozialwissenschaften an der Goethe-Universität Frankfurt am Main. Anschließend Post-Doc an der Heidelberg School of Education (Universität und Pädagogische Hochschule Heidelberg). 